\title{
DIE OKINAWA-FRAGE
}

\author{
Zur Verfassung der südjapanischen Inseln \\ Von Dr. M. Y. CHо
}

\begin{abstract}
Während uns die Teilung Deutschlands, Koreas, Vietnams und Chinas mehr oder minder bekannt ist, ist über die Teilung Japans bisher kaum etwas Wesentliches berichtet worden. Selbst in Japan sind sich nur wenige Menschen dessen bewußt, daß ihr Heimatland geteilt ist. Tatsache ist dennoch, daß infolge des Zweiten Weltkrieges der in der Hauptsache südlich des 27. (ursprünglich 29.) Breitengrades $z$ wischen dem japanischen Festland und Taiwan liegende Teil des japanischen Staatsgebietes noch immer amerikanisch „verwaltet" wird, während seine Einwohner juristisch nach wie vor japanische Staatsangehörige sind ${ }^{1}$. Die Amerikaner üben sämtliche Hoheitsrechte über die südjapanischen Inseln mit fast einer Million Einwohner aus, die bis zum Kriegsende hauptsächlich eine japanische Präfektur („Ken“) bildeten und die man heute schlechthin Okinawa zu nennen pflegt. (Okinawa ist in Wirklichkeit nur die größte und wichtigste Insel der Nansei-Inselgruppe.) So kommt es, daß kein Geringerer als James V. Martin jr., der politische Berater beim Hohen Kommissar der USA, dem Verfasser in einem Gespräch in der Hauptstadt Okinawas, Naha, gegen Ende Oktober 1965 mit aller Selbstverständlichkeit erklären konnte: „Wir müssen hier in Okinawa bleiben. Wo sonst könnten wir solche absoluten Hoheitsrechte und Bewegungsfreiheit genießen?"

Das Eigenartige an der japanischen Teilung besteht darin, daß sie nicht eine Folge der in der Weltpolitik herrschenden Spannung zwischen Ost und West ist, wie in den anderen geteilten Ländern, sondern die Spannung zwischen zwei Verbündeten widerspiegelt: nämlich $z$ wischen Japan und den Vereinigten Staaten von Amerika. Auch darin liegt wohl ein Grund dafür, daß über die Okinawa-Frage gern geschwiegen worden ist, obwohl sie keineswegs unwichtig ist. Die Bedeutung der Frage in der internationalen Politik wird uns klar, wenn wir bedenken, daß die Entwicklung der japanisch-amerikanischen Beziehungen ein entscheidender Faktor für den Erfolg oder Mißerfolg der Asien-Politik Washingtons ist. Denn ohne die Mitwirkung Tokios an der Gestaltung der amerikanischen Asien- und insbesondere ChinaPolitik würden die USA in Asien beträchtlich isoliert werden.
\end{abstract}

\section{Die Geschichte der südjapanischen Inseln bis zum Ende des Zweiten Weltkrieges}

Zum Verständnis der Okinawa-Frage ist ein Einblick in die Geschichte der Inselgruppen wichtig.

Die südjapanischen Inseln bestehen aus zwei Hauptgruppen, der Nansei-Gruppe und der Nanpo-Gruppe sowie der Markus-Insel im Osten und dem Douglas-Riff im Süden. Die Nansei-Gruppe, die aus den Ryukyu- und den Daito-Inseln besteht, wurde zunächst vermutlich von südpazifischen Siedlern entdeckt; die nachfolgenden Siedler jedoch stammten vorwiegend aus China und Japan. Von diesen beiden großen Nachbarländern wurde die Geschichte der Inseln weitgehend geformt ${ }^{2}$.

\footnotetext{
1 Vgl. dazu Saburo Kuwada, Status of Okinawans under the Japanese Nationality Law, in: Japantse Annual of International Law, 1959, S. $87 \mathrm{ff}$.; Keishin Sunagawa, The Ryukyuwans Personal Law, in:

- Japanese Annual of International Law, 1966, S. $46 \mathrm{ff}$. Okinawa: The History of an Island People, Rutland, Vermont/Tokio, Japan, 1964.
} 
Unter der Ming-Dynastie wurden im Rahmen der chinesischen Expansionspolitik von schwachen Nachbarstaaten, unter ihnen auch von Liu Ch'iu, wie die Ryukyus von China genannt wurden, Tributzahlungen gefordert. Die Ryukyus zahlten ab 1372 ungefähr fünf Jahrhunderte lang Tribute an China. $\mathrm{Zu}$ der Zeit, als die Zahlungen begannen, bestand Okinawa aus drei rivalisierenden Königreichen dem nördlichen, dem mittleren und dem südlichen - ungefähr ab 1429 wurde das Inselreich vom mittleren Königreich (Chuzan) beherrscht. Die Tributleistungen an China waren den Okinawanern nicht lästig, denn sie hielten sich in mäßigen Grenzen; die chinesische Oberheit war kaum spürbar, und die Vereinbarung brachte Handelsvorteile. Der chinesische Einfluß war von 1372 bis zur japanischen Eroberung im Jahre 1609 vorherrschend.

Okinawa stand auch nach der Eroberung durch Japan weiterhin in Tributabhängigkeit zu China, was Handelsvorteile brachte. Die Okinawaner übernahmen viele Kulturgüter von China, und chinesische Kaufleute ließen sich auf Okinawa, besonders in Naha, nieder.

Vom Zeitpunkt der Einigung der Inseln im Jahre 1429 durch Sho Hashi bis zum Ende der Regierungszeit Sho Shins 1526 genossen die Okinawaner eine Periode des Friedens, Wohlstands und kulturellen Fortschritts, die als das Goldene Zeitalter der ryukyuanischen Geschichte bekannt ist.

Andererseits begann das okinawanische Königreich aber bereits zu Beginn des 15. Jahrhunderts, auch formelle Beziehungen zu Japan aufzunehmen. Es wurden Vertreter des Königreichs mit Geschenken nach Kyoto geschickt. Ihr Weg führte sie durch Satsuma, die südlichste Provinz Japans, und das japanische Shonugat, das die Geschenke als "Tribute“ betrachtete, übertrug 1441 dem Lehnsherrn von Satsuma die Gerichtshoheit über die Ryukyus. Diese Gerichtshoheit war zunächst rein theoretisch, und Kyoto machte keinen Versuch, sich in die inneren Angelegenheiten des Inselreiches einzumischen. Im Jahre 1609 aber marschierte der Lehnsherr von Satsuma in das Inselreich ein und eroberte alle Inseln einschließlich Okinawas unter dem Vorwand, daß Okinawa während des koreanischen Krieges (1592-1598) Japan nicht unterstützt habe. Die Eroberung wurde durch ein schriftliches Abkommen formalisiert, in dem festgestellt wurde, daß die Inseln „von alters her zu Satsuma im Feudalverhältnis gestanden haben " 3 .

Damit war die Vorherrschaft des japanischen Einflusses endgültig befestigt und die des chinesischen ausgeschaltet. China setzte sich nicht zur Wehr, und rund 260 Jahre lang wurden die außen- und innenpolitischen Angelegenheiten der Ryukyus von den Satsuma-Lehnsherren überwacht. 1879 schaffte Japan die Monarchie für die Ryukyus ab und machte die Inseln zu einer japanischen Präfektur, die der Leitung japanischer, von Tokio ernannter Beamten unterstand. Die Ryukyuaner wehrten sich nicht gegen diese Annektion, weil sie ihnen auch Vorteile brachte. Der einheimische Dialekt wurde durch Japanisch abgelöst, und dadurch wurde die Errichtung eines Schulwesens möglich. Die Zugehörigkeit zu einem kräftigen und sich ausbreitenden Imperium schien außerdem politische Stabilität und Frieden zu gewährleisten. Dieser wurde mit dem Zweiten Weltkrieg unterbrochen.

Der südliche Teil der Nanpo-Gruppe, der aus den Bonin-Inseln, den VulkanInseln und der Insel Rosario besteht, ist gegenüber der Nansei-Gruppe mit den Ryukyu-Inseln verhältnismäßig bedeutungslos. Er war im Jahre 1944 von knapp 8000 Menschen bewohnt, unter denen Europäer japanischer Staatsangehörigkeit

3 Zitiert in: Yoshio Kuno, Japanese Expansion on the Asiatic Continent, Berkeley 1949, Bd. 2, S. 288-293. 
eine große Gruppe bildeten. Verwaltungsmäßig unterstand er der Präfektur Tokio. Kurz vor dem Ende des Zweiten Weltkrieges wurde die gesamte Bevölkerung auf das japanische Festland evakuiert.

\section{Die Geschichte der südjapanischen Inseln seit dem Ende des Zweiten Weltkrieges}

Die Geschichte der südjapanischen Inseln seit dem Zweiten Weltkrieg ist zugleich die der Entwicklung der japanisch-amerikanischen Beziehungen und somit die der sogenannten Okinawa-Frage. Vier Phasen sind deutlich erkennbar.

Die erste Periode begann am 1. April 1945 mit der Landung der 10. US-Armee unter dem Kommando von Generalleutnant Simon Bolivar Buckner auf Okinawa. Für die US-Armee war die Insel von großer Bedeutung als militärischer Stützpunkt gegen das japanische Festland. Im Laufe einer 83tägigen Schlacht, der letzten im Zweiten Weltkrieg und der größten im pazifischen Raum, fielen 90000 japanische Kampftruppen, über 100000 japanische Zivilisten und 11400 amerikanische Soldaten, darunter der Oberbefehlshaber Buckner. Der Oberbefehlshaber der 32. japanischen Armee, General Mitsuru Ushijima nahm sich zusammen mit seinem Stabschef, Isamu Cho, das Leben, als die Schlacht am 22. Juni 1945 mit einer totalen Niederlage der Japaner endete4. Damit war die Basis für die Bombardierung des japanischen Festlandes geschaffen. Am 15. August, drei Wochen später, kapitulierte Japan bedingungslos, womit die Periode der Feindseligkeiten in den japanisch-amerikanischen Beziehungen beendet war.

Die danach folgende zweite Periode war dadurch charakterisiert, daß den südjapanischen Inseln, insbesondere den Ryukyu-Inseln, keine Aufmerksamkeit mehr geschenkt wurde, da sie ihre militärische Bedeutung als Stützpunkt gegenüber dem japanischen Festland verloren hatten. Die Vereinigten Staaten gestatteten allerdings nur 135 Einwohnern europäischer Herkunft die Rückkehr auf die südlichen Inseln der Nanpo-Gruppe. Die Nansei-Gruppe verloren die USA praktisch aus den Augen. Das Oberkommando in Tokio, das die Verantwortung für die Inseln trug, ernannte Außenposten für Okinawa. Militärs betrachteten die Insel als eine Art Verbannungsort, und für strebsame Beamte war Okinawa ein „Niemandsland“, „das Ende der Welt", oder eine Art Sibirien ${ }^{5}$. Nur wenige von Format oder verwaltungstechnischem Geschick fanden sich bereit, in Okinawa zu bleiben. Es herrschte eine erschreckende Gleichgültigkeit diesen Inseln gegenüber ${ }^{6}$. Auch die nationalchinesische Forderung vom 18. Oktober 1947, „Okinawa an China zurückzugeben“, brach diese Indifferenz nicht. Sie sollte andauern, bis ein offizieller Friedensvertrag mit Japan die japanische Verwaltung auf den Inseln wiederherstellen und den Abzug der amerikanischen Truppen von den Inseln ermöglichen würde. Die schnelle Entwicklung der weltpolitischen Ereignisse im Fernen Osten führte jedoch dazu, daß diese Periode der Vergessenheit Okinawas überraschenderweise schon innerhalb von vier Jahren ein Ende nahm.

Während der dritten Periode, die durch die Ausrufung der Volksrepublik China am 1. Oktober 1949 eingeleitet und danach durch die Entstehung der chinesischsowjetischen Solidarität und die Zuspitzung des kalten Krieges gekennzeichnet wurde, gewann Okinawa wieder an Bedeutung als militärischer Stützpunkt - dies-

- S. Takushiro Hattori, Dai-to a Zenshi (Die vollständige Geschichte des Großostasienkrieges), Tokio 1965, S. $808-809$

5 S. Lawrence Olson, Dimensions of Japan, New York 1963, S. 362.

- George H. Kerr, a. a. O., S. 5. 
mal aber gegenüber dem chinesischen Festland und der kommunistischen Expansion im Fernen Osten überhaupt ${ }^{7}$. Die erfolgreiche Benutzung Okinawas als Stützpunkt während des Korea-Krieges bestätigte überzeugend die militärische Bedeutung der Insel.

Im Dezember 1950 wurden der neugeschaffenen US-Verwaltung der RyukyuInseln (United States Administration of the Ryukyu-Islands - USCAR) alle Aufgaben der Militärverwaltung für die Nansei-Gruppe übertragen. Der Oberkommandierende Fernost wurde zum Gouverneur, der kommandierende General der Ryukyus zum stellvertretenden Gouverneur ernannt. Am 1. April 1951 gestattete die USCAR die Schaffung einer provisorischen Zentralregierung, deren Chief Executive (Shuseki) vom Hohen Kommissar ernannt wurde. Seit dem 1. April 1952 heißt sie "Government of the Ryukyu-Islands“.

Ein umfassendes Programm für den Ausbau von Militärstützpunkten überschattete 1950 sofort die Pläne für den zivilen Wiederaufbau Okinawas. Alle Mittel und Energien mußten den aktuellen militärischen Anforderungen untergeordnet werden. Verantwortungsbewußte Beamte taten für die Verwaltung der Insel ihr Bestes, aber mit einem unzureichenden Budget.

Während der Vorverhandlungen zur Konferenz von San Franzisko wurden die voraussichtlichen Vertragsbedingungen in der Offentlichkeit diskutiert. Da Okinawa eine japanische Präfektur gewesen war, erwarteten sowohl die Okinawaner als auch die Japaner, daß die Inseln wieder der japanischen Verwaltung unterstellt werden würden und daß die Frage der Militärstützpunkte ebenso behandelt werde wie die der anderen ausländischen Stützpunkte in Japan. Man erwartete, daß in dem abzufassenden Dokument die Bedingungen für eine Rückkehr zu Japan und der Termin der Ablösung der amerikanischen Militärverwaltung über die Zivilbevölkerung und die Wirtschaft genannt werden w ẅrden ${ }^{8}$.

Der Vertrag, der schließlich am 8. September 1951 unterzeichnet wurde, enthielt jedoch den folgenden Artikel 3:

"Japan wird jedem Vorschlag der Vereinigten Staaten an die Vereinten Nationen zustimmen, die Nansei-Inselgruppe südlich des 29. nördlichen Breitengrades (die die Ryukyu-Inseln und die Daito-Inseln umfaßt), die Nanpo-Inselgruppe südlich von Sofu Gan (die die Bonin-Inseln, die Rosario-Inseln und die Vulkan-Inseln umfaßt) sowie Parece Vela und die Markus-Insel deren Treuhandschaft zu unterstellen, und zwar mit den Vereinigten Staaten als alleiniger Verwaltungsmacht ... Bis zu einem solchen Vorschlag und bis zur positiven Aktion in dieser Hinsicht haben die Vereinigten Staaten das Recht, alle und jegliche Gewalt der Verwaltung, Gesetzgebung und Rechtsprechung über das Gebiet und die Einwohner dieser Inseln, einschließlich ihrer territorialen Gewässer, auszuüben."

Aus diesem Artikel geht nicht nur hervor, daß eine völkerrechtliche Basis für die amerikanische Ausübung der Verwaltung über die südjapanischen Inseln geschaffen war, sondern auch, daß die amerikanische Verwaltung eigentlich nichts anderes sein sollte als eine Übergangsmaßnahme, bis das Treuhandsystem auf die Inseln angewandt würde ${ }^{9}$. Erst dann sollte Japan formell seine Hoheitsrechte über die Inseln verlieren, da die Potsdamer Erklärung vom 26. Juli 1945 besagte: „8. Wie in der Proklamation von Kairo ausgeführt wird, wird Japans Souveränität auf die Inseln

\footnotetext{
; James Cary, Japan Today: Reluctant Ally, New York 1962, S. 189.

${ }^{8}$ Vgl. Mikio Higa, Okinawa: Seiji to Seito („Okinawa: Politik und Parteien“), Tokio 1965, S. 7-3 und S. 66.

9 Vgl. Yoshio Nakano u. a., Okinawa Mondai 20 Nen („20 Jahre Okinawa-Frage“), Tokio 1965, S. 46; vgl. dazu Survey of International Affairs 1949-1950, Oxford University Press 1953, S. 345.
} 
Hondo, Hokkaido, Kyushu, Shikoku und die von uns noch zu bestimmenden kleinen Inseln beschränkt.“

\section{Die Auseinandersetzung um Artikel 3 des Friedensvertrages mit Japan}

Eine genauere Analyse des Artikels 3 im Friedensvertrag läßt erkennen, daß die eigentliche Bedeutung des Artikels darin liegt, daß er „der amerikanischen Regierung erlaubte, eine richtiggehende Annektion der Ryukyus zu umgehen, aber alle Rechte und Gewalt zu erhalten, die eine Anektion mit sich gebracht hätte"10. In diesem Zusammenhang wies der gebürtige Okinawaner Higa, Professor der politischen Wissenschaft an der Universität Ryukyu, in einem Gespräch mit dem Verfasser vor allem auf die Tatsache hin, daß der in Artikel 3 vorgesehene Vorschlag der USA an die UN bezüglich des Treuhandsystems nie gemacht worden ist ${ }^{11}$. Dieses Versäumnis ist insofern verständlich, als für die Anwendung des Treuhandsystems auf die südjapanischen Inseln die USA entweder die Genehmigung des Weltsicherheitsrates (Artikel 82, 83 der Satzung der Vereinten Nationen) und damit die Zustimmung der UdSSR benötigen oder nach der Genehmigung der Generalversammlung der UN mit der periodischen Aufsicht des Treuhandschaftsrates, dem auch die Sowjetunion in ihrer Eigenschaft als ständiges Mitglied des Weltsicherheitsrates angehört, rechnen müßten.

Völkerrechtlich ist es nicht klar, ob die Inseln überhaupt als „Treuhandgebiet“ gelten könnten. Das Treuhandsystem kann im allgemeinen angewandt werden

1. auf alle Gebiete, die früher Mandate des Völkerbundes waren,

2. auf Gebiete, die nach dem Zweiten Weltkrieg von ehemaligen Feindstaaten der

Siegermächte abgetrennt wurden, und

3. auf Gebiete, die von den für die Verwaltung verantwortlichen Staaten dem Treuhandschaftssystem freiwillig unterstellt werden (Artikel 77 SVN).

Die Ryukyu-Inseln könnten höchstens unter die zweite der drei Kategorien von Treuhandgebieten fallen. Darauf wies auch Dulles hin ${ }^{12}$.

Inzwischen war in Japan und in anderen Staaten die Kritik an dieser Politik der USA wach geworden. Und Dulles versuchte, ihr mit der sogenannten Doktrin der "Residual Sovereignty“, die er selbst auf der Friedenskonferenz von San Franzisko im Jahre 1951 formulierte, zu begegnen. Danach blieb die japanische Hoheit über die Ryukyu-Inseln erhalten, so daß die Vereinigten Staaten die südjapanischen Inseln nicht mehr einer Treuhandschaft der Vereinten Nationen unterstellen konnten und schließlich die Inseln wieder der japanischen Verwaltung unterstellen müßten. Aus der „Residual Sovereignty“ folgern die Vereinigten Staaten auch, daß die Einwohner der Inseln japanische Staatsbürger geblieben sind und nicht die Rechte der Einwohner amerikanischer Territorien, wie etwa Puerto Ricos, erworben haben ${ }^{\mathbf{1 3}}$.

Die Vereinigten Staaten demonstrierten die Bedeutung des Begriffes der japanischen "Residual Sovereignty“ durch die Rückgabe von Amami-Oshima, eines Teils der Ryukyu-Inselgruppe, an Japan am 25. Dezember 1953. Die Rede von William Sebald vor der amerikanisch-japanischen Gesellschaft in Tokio am 28. September

\footnotetext{
10 Mikio Higa, a. a. O., S. 69.

11 Vgl. dazu U.S. Army Area Handbook for Japan, Washington 1961, S. 520 ff.

12 Mikio Higa, a a O, S. 69.

13 Vgl. S. Kuwada, Status of Okinawans under the Japanese Nationality Law, in: The Japanese Annual of International Law 1959, S. $87 \mathrm{ff}$.
} 
$1951^{14}$ und die gemeinsamen Kommuniqués von Eisenhower und Kishi 195715 und von Kennedy und Ikeda $1961^{16}$ bestätigten die japanische „Residual Sovereignty“. Schließlich betonen die japanischen Völkerrechtler im allgemeinen einen Unterschied zwischen Gebietshoheit und Personalhoheit. Sie behaupten, daß Japan nicht alle Rechte und Ansprüche auf die Inseln aufgegeben, sondern lediglich den Vereinigten Staaten das Recht eingeräumt habe, die Inseln zu verwalten. Daher kann über dieses Territorium ohne die Zustimmung Japans nicht einseitig durch die Vereinigten Staaten verfügt werden ${ }^{17}$. Es handelt sich also ähnlich der deutschen Interpretation der Rechtsstellung Polens und der Sowjetunion in den deutschen Ostgebieten nur um eine Verwaltungszession und nicht um eine Gebietszession. Anläßlich der Rückgabe von Amami-Oshima an Japan erklärte Dulles 1953, daß die Vereinigten Staaten so lange „Vormund“ der südlichen Ryukyu-Inseln bleiben würden, „solange die Bedrohung und Spannung im Fernen Osten anhält" ${ }^{18}$. Bemerkenswert ist, daß hier nicht mehr von der Möglichkeit einer Treuhandschaft die Rede war, sondern daß die amerikanische Oberhoheit als „Vormundschaft" aufgefaßt wurde. Das bedeutete, daß die Amerikaner zwar für unbegrenzte Dauer, aber nicht mehr für immer auf Okinawa zu bleiben gedachten. In diesem Sinne schien die amerikanische OkinawaPolitik und damit die Auslegung des Artikels 3 des Friedensvertrages, in dem die Okinawa-Frage im eigentlichen Sinne verwurzelt ist, mehr oder minder geklärt worden zu sein.

Völlig unerwartet rückte Außenminister Dulles im August 1956 die Okinawa-Frage in ein ganz neues Licht. Er deutete an, daß in dem Falle, daß Japan sich bei seinen Bemühungen um einen formellen Frieden mit der Sowejtunion mit der ständigen Besetzung gewisser Inseln der Südlichen Kurilen durch die Sowjetunion einverstanden erklären werde, die Vereinigten Staaten die Doktrin der „Residual Sovereignty", die er 1951 und 1953 formuliert hatte, wieder in Frage stellen müßten. Mit anderen Worten könnten die Vereinigten Staaten immer noch entscheiden, die Ryukyus für immer zu behalten ${ }^{19}$. Okinawa war nun von großem Wert als Verhandlungsobjekt gegenüber der Sow jetunion und Japan. Der Wunsch der Okinawaner nach Rückkehr zu Japan wurde völlig ignoriert. Immerhin wurde im Jahre 1957 endlich wenigstens die Rechtsstellung der Verwaltung für die NanseiGruppe festgelegt und geordnet ${ }^{20}$. Die Verwaltung der übrigen Inseln, auf denen heute keine Japaner leben, ist, soweit ersichtlich, nirgendwo geregelt worden. Auch der amerikanische Conlon-Bericht vom 1. November 1959, der die Rückgabe Okinawas an Japan befürwortete, zeitigte keine konkreten Ergebnisse. Schließlich erwies sich das Treffen $z$ wischen Präsident Kennedy und Ministerpräsident Ikeda in Washington im Juni 1961, auf das die Okinawaner im Hinblick auf die Rückkehr zu Japan so viel Hoffnung gesetzt hatten, als ergebnislos. So blieb diese dritte Periode weiterhin eine Zeit der Spannung.

Die vierte und gegenwärtige Periode der Entwicklung der Okinawa-Frage wurde durch eine Erklärung des US-Präsidenten Kennedy am 19. März 1962 eingeleitet, in der er die Ryukyu-Inseln of fiziell als „Bestandteil des japanischen Mutterlandes“ anerkannte und ankündigte, daß eine Reihe von Maßnahmen ergriffen werden sollte, „um die Schwierigkeiten zu vermindern, die die letzten Endes vorgesehene

\footnotetext{
14 Okinawa no Chii (Die Stellung Okinawas), herausgegeben von der Japanischen Gesellschaft für Völkerrecht,

15 The New York Times, 22. Juni 1957.

16 The New York Times, 23. Juni 1961.

17 Okinawa no Chii, S. 70-71, 102-105 und 110-112.

18 The New York Times, 25. Dezember 1953.

19 Vgl. George R. Packard III, Protest in Tokyo, Princeton University Press 1966, S. 71-72.

20 Exekutive Order 10713 vom 5. Juni 1957, geändert am 19. März 1962.
} 
Rückgabe der Ryukyu-Inseln an die japanische Verwaltung begleiten werden" ${ }^{21}$. Von den südlichen Inseln der Nanpo-Gruppe und den beiden abgelegenen Inseln war bei dieser Gelegenheit wie auch in allen früheren und späteren Äußerungen weder von amerikanischer noch von japanischer Seite die Rede.

Die Kennedy-Erklärung drückte die Absicht der Vereinigten Staaten aus, „die militärische Notwendigkeit der Fortsetzung der amerikanischen Verwaltung mit dem Wunsch der Ryukyu-Bevölkerung, die sich zu Japan gehörig fühlt “22, zu vereinbaren. Sie bedeutete ferner eine weitere Klärung der amerikanischen Stellung auf Okinawa, indem die „Residual Sovereignty“ Japans über die Ryukyu-Inseln dabei ganz ausdrücklich bestätigt wurde. Die Erklärung wurde daher als die Ankündigung einer neuen Okinawa-Politik der Vereinigten Staaten betrachtet ${ }^{23}$. Völkerrechtlich gesehen lag die Bedeutung der Kennedy-Erklärung darin, daß Washington ausdrücklich die Absicht aufgab, je den Vereinten Nationen die Treuhandverwaltung der Inseln vorzuschlagen. Daraus folgt, daß der Artikel 3 des Friedensvertrages zwar nicht seine Gültigkeit, aber seine politische Basis verloren hat. Von japanischer Seite wird daher gefordert, in neuen Verhandlungen die Verwaltung der Inseln unter Berücksichtigung der amerikanischen und zugleich japanischen militärischen Interessen zu regeln ${ }^{24}$.

Der Kennedy-Erklärung folgte keine sehr „neue“ Politik in Okinawa. Es wurde zwar am Tage der Erklärung die Verwaltungsordnung dahin ergänzt, daß die Bevölkerung ein Parlament wählen sollte, aber die Verwaltung der Inseln sollte diesem nicht verantwortlich sein ${ }^{25}$. Das Parlament hatte also nur beratende Funktion. Immerhin ist seitdem doch wenigstens ein gewisser Annäherungsprozeß zwischen Japan und den USA unverkennbar im Gange. So besuchte bereits drei Monate nach der Erklärung zum ersten Male eine Delegation der japanischen Regierung Okinawa, um sich dort über die allgemeine Lage zu informieren. Am 2. November 1962 wurde in einem Gespräch zwischen dem japanischen Außenminister Ohira und dem amerikanischen Botschafter Reischauer die Gründung eines japanisch-amerikanischen Beratungskomitees für die Okinawa-Hilfe mit Sitz in Tokio und eines amerikanischjapanisch-ryukyuanischen technischen Komitees mit Sitz in Okinawa beschlossen. Das. erste Treffen des Beratungskomitees fand am 25. April 1964 statt; das erste Treffen des technischen Komitees am 15. Juli 1964. Am 1. August 1964 trat Generalleutnant Albert Watson II das Amt des Hohen Kommissars der USA in Okinawa an. Er kündigte an, er würde im Sinne der "neuen Okinawa-Politik“ des verstorbenen Präsidenten Kennedy eng mit der Bevölkerung Okinawas zusammenarbeiten ${ }^{26}$. In dem gemeinsam von Präsident Johnson und Premierminister Sato in Washington am 13. Januar 1965 veröffentlichten Kommuniqué hieß es unter anderem: „In Anerkennung des Wunsches der Regierung und des Volkes von Japan erklärte der Präsident, daß er sich auf den Tag freue, an dem das Interesse der Sicherheit der Freien Welt und des Fernen Ostens die Erfüllung dieses Wunsches ermögliche. “27

„Was den Zeitpunkt der Rückgabe betrifft“, erklärte in diesem Zusammenhang der Hohe Kommissar Watson, „so sehe ich keine Möglichkeit, was die Festlegung eines bestimmten Tages für die Rückgabe der Verwaltung betrifft. Ich weiß nicht, wann

\footnotetext{
21 The New York Times, 20. März 1962, vgl. Roger Bersihand, Geschichte Japans, Stuttgart 1963, S. 603. 22 Ebenda.

23 Vgl. Asahi-Nenkan ( Asahi-Jahrbuch“), Tokio 1963, S. 304.

24 Vgl. R. Taoka, Legal Status of Okinawa, in: Japanese Annual of International Law 1958, S. 103.

25 Executive Order 11010 vom 19. März 1962.

26 S. Asahi-Nenkan 1965, S. 296 und 564.

27 Vgl. dazu den Beitrag d. Verf. „Japans asiatische Verantwortung ${ }^{\alpha}$, in: Außenpolitik 1965, Bd. 12, S. 835.
} 
die bestehenden Bedrohungen und Spannungen soweit nachlassen, daß die Streitkräfte der Vereinigten Staaten ohne Gefahr nach Japan, den Vereinigten Staaten und anderen Ländern abgezogen werden könnten. Die Bedrohungen und Spannungen und der gegenwärtige Konflikt sind nicht in Washington, Tokio oder Naha, sondern in Peking, Hanoi und Pyongyang entstanden ...

Die Vereinigten Staaten verwalten die Ryukyu-Inseln, weil sie in der Lage sein müssen, schnell und wirksam ihre Verpflichtungen für die Verteidigung Japans und anderer Länder der Freien Welt im Fernen Osten zu erfüllen. Die Fähigkeit, unseren Verpflichtungen nachzukommen, erfordert, daß wir für unsere Streitkräfte einen Stützpunkt zur Verfügung haben, auf dem und von dem aus wir Handlungsfreiheit haben ...

Der Verlust von Verwaltungsrechten würde die militärische Handlungsfreiheit unserer Streitkräfte vermindern oder ausschalten und würde die Nutzbarkeit Okinawas als eines Stützpunktes für die Verteidigung der Interessen der Freien Welt, einschließlich Japans, ernsthaft schwächen ...

Die Frage zwischen den Vereinigten Staaten und den Ryukyuanern oder den Japanern ist nicht, ob die Rückgabe erfolgen soll. Die Schwierigkeit liegt darin, daß es jetzt nicht möglich ist, zu entscheiden, wann'."28

Damit machen sich die Vereinigten Staaten im Grunde die These der sowjetischen Völkerrechtslehre zu eigen, daß strategische Erfordernisse eine Vernachlässigung des Selbstbestimmungsrechts rechtfertigen ${ }^{29}$.

Die japanische Regierung ihrerseits stellte auf der 2. Kabinettsberatung über die Okinawa-Fragen am 7. September 1965 fest, daß 1. die japanische Verfassung in Okinawa abstrakt praktiziert, aber nicht konkret angewandt wird, 2. es keinen Zusammenhang zwischen der amerikanischen Verwaltung Okinawas auf Grund des Artikels 3 des Friedensvertrages und den Bestimmungen über das Treuhandsystem im Artikel 78 der Satzung der Vereinten Nationen gibt und 3. die Frage der Rückgabe Okinawas nur durch ein freundschaftliches Ubereinkommen zwischen Tokio und Washington gelöst und nicht vor die Weltorganisation gebracht werden solle ${ }^{30}$.

Mit dieser Feststellung wurde nicht nur eine juristische Formel über den Status der Ryukyu-Inseln aus japanischer Sicht geschaffen, sondern es wurde damit auch offiziell die „Residual Sovereignty" Japans über Okinawa nochmals bekräftigt, die Fortsetzung der amerikanischen Verwaltung in Okinawa als militärische Notwendigkeit bestätigt und die Okinawa-Frage als eine rein japanisch-amerikanische Angelegenheit betrachtet. Der gute Wille der USA in der Okinawa-Frage kam deutlich zum Ausdruck, als am 20. Dezember 1965 von der amerikanischen Regierung beschlossen wurde, daß der Chief Executive der Regierung der RyukyuInseln nicht mehr vom Hohen Kommissar ernannt, sondern durch das ryukyuanische Parlament gewählt werde solle. Dieser Schritt wurde überall in Naha und Tokio als ein Fortschritt begrüß $\mathrm{t}^{31}$.

Die Entwicklung der japanisch-amerikanischen Beziehungen in der Okinawa-Frage seit 1966 zeigt, daß beide Länder bemüht sind, soweit wie möglich sich gegenseitig anzupassen, auch wenn in militärischer Hinsicht der Status quo erhalten bleiben muß. Wir können also diese Periode eine Periode der Annäherung nennen.

\footnotetext{
28 Albert Watson, The United States in the Ryukyu Islands, (Vortrag vor dem Foreign Correspondents Club

29 Vgl. B. Meissner, Sowjetunion und Selbstbestimmungsrecht, Köln 1962, S. 66.

so Vgl. Kagami, III. Band, 3. Heft 1965, herausgegeben vom Institut für Asienkunde in Hamburg und der Deutschen Geselischaft für Natur- und Völkerkunde Ostasiens in Tokio, S. 115.

s1 Vgl. dazu Asahi Shimbun, 22. Dezember 1965.
} 
Man denke dabei beispielsweise nur an den sogenannten „Mori-Plan“ für die Rückgabe der Kulturverwaltung über Okinawa an Japan. Bei dem Plan, den der unter der Schirmherrschaft des Verwaltungsdirektors Mori stehende Rat für die Diskussion der Okinawa-Frage entwickelt hat, geht man davon aus, daß es völkerrechtlich möglich ist, die Kulturverwaltung von den amerikanischen Verwaltungsrechten über die Ryukyu-Inseln zu trennen und sie an Japan zurückzugeben. Auf dem ersten Treffen des Rates am 1. September 1966 argumentierten die beiden prominenten Juristen Kisaburo Yokota und Shuzo Hayashi: „Die Trennung der Verwaltungsrechte ist möglich, wie dies aus dem Verhältnis zwischen der Bundesregierung und den Regierungen der Bundesstaaten in den USA ersichtlich ist. Bei der Rückübertragung der Kulturverwaltung an die japanische Regierung entstehen daher juristisch keinerlei Nachteile für die amerikanischen Verwaltungsrechte. " 32

Könnte man dabei sogar an die Kulturhoheit der Länder in der Bundesrepublik Deutschland erinnern, um die Trennung der Hoheitsrechte zwischen Bund und Ländern zu veranschaulichen, so würde die Rückgabe der Kulturverwaltung über die Ryukyu-Inseln an Japan noch einen weiteren Beweis für die Unhaltbarkeit des Artikels 3 des Friedensvertrages bedeuten. Damit würde auch dem Verbleiben der amerikanischen Truppen auf Okinawa immer mehr ein ausschließlich militärischer Charakter verliehen, dem ein Stützpunktabkommen am ehesten gerecht werden würde. Neuerdings spricht man immer mehr von der Rückgabe der adminıstrativen Rechte Japans, während die USA ihre Militärstützpunkte auf Okinawa behalten.

Die Zukunft der südjapanischen Inseln, die die Amerikaner „Grundpfeiler des Pazifiks" (the Keystone of the Pacific) nennen, hängt in der Tat von der weltpolitischen Entwicklung im pazifischen Raum ab.

Solange der Krieg in Vietnam weitergeht, werden die USA Okinawa nicht aufgeben. „Okinawa ist als ein wichtiger amerikanischer Stützpunkt im Westpazifik in die neuerliche amerikanische militärische Eskalation in Südvietnam einbezogen worden. Eine Abwehr-Raketen-Einheit, Marinetruppen und Fallschirmjäger sind von Okinawa nach Südvietnam entsandt worden. Transportschiffe laufen im Hafen von Naha ein und aus. Die Flugplätze schwirren von landenden und startenden Flugzeugen. “33

Andererseits scheinen stufenweise Konzessionen der Amerikaner zugunsten der japanischen Verwaltung und der japanischen Souveränität auf den Inseln unver. meidlich zu sein. Daß seit dem 1. Juli 1967 die japanische Nationalflagge auf den Schiffen Okinawas mit wehen darf, ist aufschlußreich in dieser Hinsicht. Auch das Ergebnis des Sato-Johnson-Gesprächs in Washington Mitte November 1967, das zwar keinen Termin für die Rückgabe Okinawas, aber immerhin die sofortige Rückgabe der Bonin-Inseln in Aussicht gestellt hat, ist eher als positiv denn als unbedeutend zu bewerten.

\footnotetext{
32 S. Tokyo Shimbun, 2. September 1966.

ss James V. Martin Jr., Vietnam and Okinawa in Perspective (Vortrag am 24. Mai 1965), in: News Release, herausgegeben vom Public Affairs Department, USCAR, Naha, 24. Mai 1965.
} 